\title{
Nonlinear Evolution Equations for Broader Bandwidth Wave Packets in Crossing Sea States
}

\author{
S. Debsarma, ${ }^{1}$ S. Senapati, ${ }^{2}$ and K. P. Das ${ }^{1}$ \\ ${ }^{1}$ Department of Applied Mathematics, University of Calcutta, 92, A.P.C. Road, Kolkata 700 009, India \\ ${ }^{2}$ Department of Mathematics, Abhedananda Mahavidyalaya, Sainthia, Birbhum 731234, India \\ Correspondence should be addressed to S. Debsarma; suma_debsarma@rediffmail.com
}

Received 14 February 2014; Accepted 2 May 2014; Published 9 June 2014

Academic Editor: Leonard Pietrafesa

Copyright (C) 2014 S. Debsarma et al. This is an open access article distributed under the Creative Commons Attribution License, which permits unrestricted use, distribution, and reproduction in any medium, provided the original work is properly cited.

\begin{abstract}
Two coupled nonlinear equations are derived describing the evolution of two broader bandwidth surface gravity wave packets propagating in two different directions in deep water. The equations, being derived for broader bandwidth wave packets, are applicable to more realistic ocean wave spectra in crossing sea states. The two coupled evolution equations derived here have been used to investigate the instability of two uniform wave trains propagating in two different directions. We have shown in figures the behaviour of the growth rate of instability of these uniform wave trains for unidirectional as well as for bidirectional perturbations. The figures drawn here confirm the fact that modulational instability in crossing sea states with broader bandwidth wave packets can lead to the formation of freak waves.
\end{abstract}

\section{Introduction}

The study of evolution of weakly nonlinear surface gravity waves in crossing sea states has attracted considerable interest in recent years. The reason is that the freak waves (freak waves are extreme oceanic waves, also known as rogue waves or giant waves or killer waves) are frequently observed in crossing sea states, characterized by two different wave-train systems. The extraordinarily large amplitude freak waves generated in crossing sea states may even lead to ship accidents (see $[1,2])$. Several mechanisms (see $[1,3,4])-$ dispersion enhancement of transient wave groups, effect of variable bathymetry, wave-current interaction, nonlinear modulational instability, statistical approach with the assumption of Gaussian wave field, and so forth-have been suggested for the possible formation of such killer waves. The problem of weakly nonlinear interaction between two wave systems propagating in two different directions has been studied by some authors ([5-7]) as a possible mechanism resulting in freak wave generation. Onorato et al. [5] derived two coupled nonlinear Schrödinger equations that describe the nonlinear interaction of two weakly nonlinear, obliquely propagating wave packets. By the use of these equations they obtained an expression for the growth rate of instability for unidirectional perturbation. Shukla et al. [6] extended their result by plotting instability growth rate diagrams for bidirectional perturbation. In both papers, stability analysis has been performed with two coupled evolution equations, correct up to third order in wave steepness. In Onorato et al. [5] and also in Shukla et al. [6], it is concluded that freak waves can be formed due to weakly nonlinear interaction in crossing sea states. But it is well known that an evolution equation, which is correct up to fourth order in wave steepness, gives a more appropriate description of the dynamical behavior of waves with sufficiently small wave steepness. The reason is that, at fourth order, the wave-induced mean flow terms appear and these terms modify considerably the growth rate of instability ([8]).

Keeping this in view, Gramstad and Trulsen [9] have derived two coupled modified nonlinear Schrödinger (hereafter referred to as CMNLS) equations that describe the evolution of two two-dimensional narrow band wave systems with different directions of propagation. The corresponding coupled evolution equations for broader bandwidth wave packets in crossing sea state condition are derived in the present paper. The importance of the assumption of broader bandwidth wave packets lies in the fact that, in reality, 
the bandwidth of the ocean wave spectra often exceeds the range of the narrow bandwidth condition. The evolution equation for a single broader bandwidth wave packet was first derived by Trulsen and Dysthe [10]. The order of the bandwidth, for narrow band wave packet, is taken as $\epsilon, \epsilon$ being a measure of weakness of nonlinearity. Trulsen and Dysthe [10] chose the order of bandwidth to be of $O\left(\epsilon^{1 / 2}\right)$ and derived the evolution equation, correct up to $O\left(\epsilon^{7 / 2}\right)$ terms. The instability regions of a uniform Stokes wave in the perturbed wavenumber plane obtained from this equation are found to be in better agreement with the exact results of McLean et al. [11]. An equation, which is one order higher than the equation derived by Trulsen and Dysthe [10], was later derived by Debsarma and Das [12], which is correct up to $O\left(\epsilon^{4}\right)$ terms. In this paper, we have presented also the fourth order nonlinear evolution equations in crossing sea states for narrow band wave packets. These equations are in a form different from those derived by Gramstad and Trulsen [9], in the sense that the two equations do not involve the wave-induced mean flow velocity potential explicitly. The two evolution equations of Gramstad and Trulsen [9] can however be rewritten as two equations that do not involve mean flow potential terms explicitly by substituting mean flow potential terms $\phi_{a}$ and $\phi_{b}$ as given in (11) of Gramstad and Trulsen [9]. The evolution equations of Gramstad and Trulsen [9] also have certain advantages because of their Hamiltonian structure. It is well known that the Hamiltonian structure provides a systematic account of symmetries inherent to the problem and of corresponding conservation laws (see [13]).

Using the two coupled nonlinear envelope equations for both narrow and broader bandwidth wave packets, we have investigated the stability of two obliquely propagating uniform wave trains. It is observed that the growth rate of instability in crossing sea states is much higher than that for a single wave packet. Expectedly, it is found that the growth rate of instability of one wave train becomes higher with the increase of the amplitude of the second wave train. We have also plotted figures showing a comparison between the growth rate of instability for narrow and broader bandwidth wave packets. It is also observed that the growth rate of instability becomes higher when the angle of interaction between two wave packets becomes smaller. Shrinkage is observed in the unstable region in the perturbed wavenumber plane when crossing sea states with broader bandwidth wave packets is considered.

\section{Derivation of Evolution Equations}

Taking $x y$-plane as the undisturbed free water surface, we consider propagation of two wave packets centered at wavenumbers $\vec{k}_{a}=(k, l)$ and $\vec{k}_{b}=(k,-l)$, respectively. Both waves have the same linear frequency $\omega_{c}=\sqrt{g k_{c}}$, where $k_{c}=\left|\vec{k}_{a}\right|=\left|\vec{k}_{b}\right|$ and $g$ is the acceleration due to gravity. The two wave packets are meeting at an angle $2 \theta$. To derive envelope equations we have followed the standard methodology that starts with Zakharov [14] integral equation in the spatial dimensions as follows:

$$
\begin{aligned}
& i \frac{\partial \mathscr{B}(\vec{k}, t)}{\partial t} \\
& =\iiint_{-\infty}^{\infty} T\left(\vec{k}, \vec{k}_{1}, \vec{k}_{2}, \vec{k}_{3}\right) \mathscr{B}^{*}\left(\vec{k}_{1}, t\right) \mathscr{B}\left(\vec{k}_{2}, t\right) \\
& \times \mathscr{B}\left(\vec{k}_{3}, t\right) \delta\left(\vec{k}+\vec{k}_{1}-\vec{k}_{2}-\vec{k}_{3}\right) \\
& \quad \times \exp \left\{i \left[\omega(\vec{k})+\omega\left(\vec{k}_{1}\right)\right.\right. \\
& \left.\left.\quad-\omega\left(\vec{k}_{2}\right)-\omega\left(\vec{k}_{3}\right)\right] t\right\} d \vec{k}_{1} d \vec{k}_{2} d \vec{k}_{3},
\end{aligned}
$$

where $\mathscr{B}(\vec{k}, t)$ is related to the free surface elevation $\zeta(\vec{x}, t)$ by the following equation:

$$
\begin{aligned}
\zeta(\vec{x}, t)=\frac{1}{2 \pi} \int_{-\infty}^{\infty} & {\left[\frac{|\vec{k}|}{2 \omega(\vec{k})}\right]^{1 / 2} } \\
& \times[\mathscr{B}(\vec{k}, t) \exp \{i[\vec{k} \cdot \vec{x}-\omega(\vec{k}) t]\} \\
& + \text { c.c. }] d \vec{k} .
\end{aligned}
$$

Here c.c. denotes complex conjugate and $\mathscr{B}^{*}=$ c.c. of $\mathscr{B}$. The kernel function $T\left(\vec{k}, \overrightarrow{k_{1}}, \overrightarrow{k_{2}}, \vec{k}_{3}\right)$ was first given by Zakharov [14] and, later on, its expression that corresponds exactly to the Hamiltonian was derived by Krasitskii [15]. Because of the presence of the factor $\delta\left(\vec{k}+\vec{k}_{1}-\vec{k}_{2}-\vec{k}_{3}\right)$ in (1) a nonzero contribution to the integral in (1) with $\vec{k}=$ $\vec{k}_{a}$ is obtained for the three cases; namely, $\left(\vec{k}_{1}, \vec{k}_{2}, \vec{k}_{3}\right)=$ $\left(\overrightarrow{k_{b}}, \vec{k}_{b}, \vec{k}_{a}\right),\left(\overrightarrow{k_{b}}, \vec{k}_{a}, \vec{k}_{b}\right)$, and $\left(\vec{k}_{a}, \vec{k}_{a}, \vec{k}_{a}\right)$. So, for the derivation of the envelope equation of first wave packet having carrier wavenumber vector $\vec{k}_{a}$ we take $\vec{k}=\vec{k}_{a}+\vec{\chi},\left(\vec{k}_{1}, \vec{k}_{2}, \vec{k}_{3}\right)=$ $\left(\vec{k}_{b}+\vec{\chi}_{1}, \vec{k}_{b}+\vec{\chi}_{2}, \vec{k}_{a}+\vec{\chi}_{3}\right),\left(\vec{k}_{b}+\vec{\chi}_{1}, \vec{k}_{a}+\vec{\chi}_{2}, \vec{k}_{b}+\vec{\chi}_{3}\right)$, and $\left(\vec{k}_{a}+\vec{\chi}_{1}, \vec{k}_{a}+\vec{\chi}_{2}, \vec{k}_{a}+\vec{\chi}_{3}\right)$. Clearly, a nonzero contribution to the integral in (1) is obtained when $\vec{\chi}+\vec{\chi}_{1}-\vec{\chi}_{2}-\vec{\chi}_{3}=0$. Similarly, for the derivation of the second envelope equation we take $\vec{k}=\vec{k}_{b}+\vec{\chi},\left(\vec{k}_{1}, \vec{k}_{2}, \vec{k}_{3}\right)=\left(\vec{k}_{a}+\vec{\chi}_{1}, \vec{k}_{a}+\vec{\chi}_{2}, \vec{k}_{b}+\vec{\chi}_{3}\right),\left(\vec{k}_{a}+\vec{\chi}_{1}, \vec{k}_{b}+\right.$ $\left.\vec{\chi}_{2}, \vec{k}_{a}+\vec{\chi}_{3}\right)$, and $\left(\vec{k}_{b}+\vec{\chi}_{1}, \vec{k}_{b}+\vec{\chi}_{2}, \vec{k}_{b}+\vec{\chi}_{3}\right)$. It is assumed that the spectral widths $|\vec{\chi}| / k_{c},\left|\vec{\chi}_{j}\right| / k_{c}$ are of order $O(\epsilon)$ for narrow band wave packet and of order $O\left(\epsilon^{1 / 2}\right)$ for broad band wave packet, $\epsilon$ being the order of smallness of wave steepness. The process of derivation of envelope equations adapted here is similar to the process of derivation of evolution equations for a surface gravity wave packet in the presence of another wave packet described in Debsarma and Das [16].

Finally, the envelope equations are written in terms of $a_{1}$ and $a_{2}$ which are the complex amplitudes of the two wave envelopes given by

$$
\begin{aligned}
& \zeta_{1}(\vec{x}, t)=\frac{1}{2}\left[a_{1}(\vec{x}, t) \exp \left\{i\left(\vec{k}_{a} \cdot \vec{x}-\omega\left(\vec{k}_{a}\right) t\right)\right\}+\text { c.c. }\right] \\
& \zeta_{2}(\vec{x}, t)=\frac{1}{2}\left[a_{2}(\vec{x}, t) \exp \left\{i\left(\vec{k}_{b} \cdot \vec{x}-\omega\left(\vec{k}_{b}\right) t\right)\right\}+\text { c.c. }\right] .
\end{aligned}
$$


Hereafter, the equations are written using the following dimensionless quantities:

$$
\begin{gathered}
\tilde{a}_{1}=k_{c} a_{1}, \quad \tilde{a}_{2}=k_{c} a_{2}, \quad \tilde{x}=k_{c} x, \\
\tilde{y}=k_{c} y, \quad \tilde{t}=\omega_{c} t,
\end{gathered}
$$

and finally $(\sim)$ notation has been dropped out.

The two CMNLS equations for broader bandwidth wave packets, correct up to order $O\left(\epsilon^{7 / 2}\right)$ in wave steepness, are the following:

$$
\begin{aligned}
& i \frac{\partial a_{1}}{\partial t}+i \beta_{1} \frac{\partial a_{1}}{\partial x}+i \beta_{2} \frac{\partial a_{1}}{\partial y}+\beta_{3} \frac{\partial^{2} a_{1}}{\partial x^{2}} \\
& +\beta_{4} \frac{\partial^{2} a_{1}}{\partial x \partial y}+\beta_{5} \frac{\partial^{2} a_{1}}{\partial y^{2}}+i \beta_{6} \frac{\partial^{3} a_{1}}{\partial x^{3}}+i \beta_{7} \frac{\partial^{3} a_{1}}{\partial x^{2} \partial y} \\
& +i \beta_{8} \frac{\partial^{3} a_{1}}{\partial x \partial y^{2}}+i \beta_{9} \frac{\partial^{3} a_{1}}{\partial y^{3}} \\
& +\left[\beta_{10} \frac{\partial^{4} a_{1}}{\partial x^{4}}+\beta_{11} \frac{\partial^{4} a_{1}}{\partial x^{3} \partial y}\right. \\
& +\beta_{12} \frac{\partial^{4} a_{1}}{\partial x^{2} \partial y^{2}}+\beta_{13} \frac{\partial^{4} a_{1}}{\partial x \partial y^{3}}+\beta_{14} \frac{\partial^{4} a_{1}}{\partial y^{4}} \\
& +i \beta_{15} \frac{\partial^{5} a_{1}}{\partial x^{5}}+i \beta_{16} \frac{\partial^{5} a_{1}}{\partial x^{4} \partial y}+i \beta_{17} \frac{\partial^{5} a_{1}}{\partial x^{3} \partial y^{2}} \\
& \left.+i \beta_{18} \frac{\partial^{5} a_{1}}{\partial x^{2} \partial y^{3}}+i \beta_{19} \frac{\partial^{5} a_{1}}{\partial x \partial y^{4}}+i \beta_{20} \frac{\partial^{5} a_{1}}{\partial y^{5}}\right] \\
& =\lambda_{1} a_{1}^{2} a_{1}^{*}+i \lambda_{2} a_{1} a_{1}^{*} \frac{\partial a_{1}}{\partial x}+i \lambda_{3} a_{1} a_{1}^{*} \frac{\partial a_{1}}{\partial y} \\
& +i \lambda_{4} a_{1}^{2} \frac{\partial a_{1}^{*}}{\partial x}+i \lambda_{5} a_{1}^{2} \frac{\partial a_{1}^{*}}{\partial y}+\lambda_{6} a_{1} \frac{\partial}{\partial x} H_{x}\left(a_{1} a_{1}^{*}\right) \\
& +\lambda_{7} a_{1} \frac{\partial}{\partial y} H_{y}\left(a_{1} a_{1}^{*}\right)+\lambda_{8} a_{1} \frac{\partial}{\partial y} H_{x}\left(a_{1} a_{1}^{*}\right) \\
& +\mu_{1} a_{1} a_{2} a_{2}^{*}+i \mu_{2} a_{2}^{*} \frac{\partial}{\partial x}\left(a_{1} a_{2}\right)+i \mu_{3} a_{1} a_{2} \frac{\partial a_{2}^{*}}{\partial x} \\
& +i \mu_{4} a_{2} a_{2}^{*} \frac{\partial a_{1}}{\partial y}+i \mu_{5} a_{1} a_{2} \frac{\partial a_{2}^{*}}{\partial y}+i \mu_{6} a_{1} a_{2}^{*} \frac{\partial a_{2}}{\partial y} \\
& +\mu_{7} a_{1} \frac{\partial}{\partial x} H_{x}\left(a_{2} a_{2}^{*}\right)+\mu_{8} a_{1} \frac{\partial}{\partial y} H_{y}\left(a_{2} a_{2}^{*}\right), \\
& i \frac{\partial a_{2}}{\partial t}+i \beta_{1} \frac{\partial a_{2}}{\partial x}-i \beta_{2} \frac{\partial a_{2}}{\partial y}+\beta_{3} \frac{\partial^{2} a_{2}}{\partial x^{2}}-\beta_{4} \frac{\partial^{2} a_{2}}{\partial x \partial y} \\
& +\beta_{5} \frac{\partial^{2} a_{2}}{\partial y^{2}}+i \beta_{6} \frac{\partial^{3} a_{2}}{\partial x^{3}}-i \beta_{7} \frac{\partial^{3} a_{2}}{\partial x^{2} \partial y}+i \beta_{8} \frac{\partial^{3} a_{2}}{\partial x \partial y^{2}}-i \beta_{9} \frac{\partial^{3} a_{2}}{\partial y^{3}} \\
& +\left[\beta_{10} \frac{\partial^{4} a_{2}}{\partial x^{4}}-\beta_{11} \frac{\partial^{4} a_{2}}{\partial x^{3} \partial y}+\beta_{12} \frac{\partial^{4} a_{2}}{\partial x^{2} \partial y^{2}}-\beta_{13} \frac{\partial^{4} a_{2}}{\partial x \partial y^{3}}\right. \\
& +\beta_{14} \frac{\partial^{4} a_{2}}{\partial y^{4}}+i \beta_{15} \frac{\partial^{5} a_{2}}{\partial x^{5}}-i \beta_{16} \frac{\partial^{5} a_{2}}{\partial x^{4} \partial y}+i \beta_{17} \frac{\partial^{5} a_{2}}{\partial x^{3} \partial y^{2}} \\
& \left.-i \beta_{18} \frac{\partial^{5} a_{2}}{\partial x^{2} \partial y^{3}}+i \beta_{19} \frac{\partial^{5} a_{2}}{\partial x \partial y^{4}}-i \beta_{20} \frac{\partial^{5} a_{2}}{\partial y^{5}}\right]
\end{aligned}
$$

$$
\begin{aligned}
= & \lambda_{1} a_{2}^{2} a_{2}^{*}+i \lambda_{2} a_{2} a_{2}^{*} \frac{\partial a_{2}}{\partial x}-i \lambda_{3} a_{2} a_{2}^{*} \frac{\partial a_{2}}{\partial y}+i \lambda_{4} a_{2}^{2} \frac{\partial a_{2}^{*}}{\partial x} \\
& -i \lambda_{5} a_{2}^{2} \frac{\partial a_{2}^{*}}{\partial y}+\lambda_{6} a_{2} \frac{\partial}{\partial x} H_{x}\left(a_{2} a_{2}^{*}\right) \\
& +\lambda_{7} a_{2} \frac{\partial}{\partial y} H_{y}\left(a_{2} a_{2}^{*}\right)-\lambda_{8} a_{2} \frac{\partial}{\partial y} H_{x}\left(a_{2} a_{2}^{*}\right) \\
& +\mu_{1} a_{2} a_{1} a_{1}^{*}+i \mu_{2} a_{1}^{*} \frac{\partial}{\partial x}\left(a_{1} a_{2}\right)+i \mu_{3} a_{1} a_{2} \frac{\partial a_{1}^{*}}{\partial x} \\
& -i \mu_{4} a_{1} a_{1}^{*} \frac{\partial a_{2}}{\partial y}-i \mu_{5} a_{1} a_{2} \frac{\partial a_{1}^{*}}{\partial y}-i \mu_{6} a_{2} a_{1}^{*} \frac{\partial a_{1}}{\partial y} \\
& +\mu_{7} a_{2} \frac{\partial}{\partial x} H_{x}\left(a_{1} a_{1}^{*}\right)+\mu_{8} a_{2} \frac{\partial}{\partial y} H_{y}\left(a_{1} a_{1}^{*}\right) .
\end{aligned}
$$

Here $H_{x}$ and $H_{y}$ represent, respectively, $x$ and $y$ components of two-dimensional Hilbert transform operator defined by

$$
\begin{aligned}
& H_{x}[f(\xi, \eta)]=\frac{1}{2 \pi} \int_{-\infty}^{\infty} \frac{(\xi-x)}{|\vec{x}-\vec{\xi}|^{3}} f(\xi, \eta) d \xi d \eta, \\
& H_{y}[f(\xi, \eta)]=\frac{1}{2 \pi} \int_{-\infty}^{\infty} \frac{(\xi-y)}{|\vec{x}-\vec{\xi}|^{3}} f(\xi, \eta) d \xi d \eta .
\end{aligned}
$$

The coefficients $\beta_{i}$ 's, $\lambda_{i}$ 's, and $\mu_{i}$ 's are given in Appendix A. When the terms within third bracket on left-hand side of (6) are omitted, the resulting equations become CMNLS equations for narrow band wave packets, correct up to fourth order in wave steepness. The coefficients $\beta_{1}, \beta_{2}, \beta_{3}, \beta_{4}, \beta_{5}, \lambda_{1}$, and $\mu_{1}$ are in agreement with the corresponding coefficients in Shukla et al. [6].

Setting $a_{2}=0$ in the envelope equation (4) we can recover the single envelope equation for a single surface gravity wave packet with carrier wavenumber $\vec{k}_{a}$.

\section{Stability Analysis}

Two obliquely propagating Stokes wave trains as obtained from envelope equations (6) are

$$
\begin{aligned}
& a_{1}=a_{10} e^{-i\left(\lambda_{1} a_{10}^{2}+\mu_{1} a_{20}^{2}\right) t} \equiv a_{1}^{(0)}, \\
& a_{2}=a_{20} e^{-i\left(\lambda_{1} a_{20}^{2}+\mu_{1} a_{10}^{2}\right) t} \equiv a_{2}^{(0)},
\end{aligned}
$$

where $a_{10}$ and $a_{20}$ are real constants.

In order to study the effect of fourth order nonlinearity on the stability properties of the above two interacting wave trains, we introduce the following infinitesimal perturbation:

$$
\begin{aligned}
& a_{1}=a_{1}^{(0)}\left(1+a_{1}^{\prime}\right), \\
& a_{2}=a_{2}^{(0)}\left(1+a_{2}^{\prime}\right) .
\end{aligned}
$$

We now substitute (9) in the envelope equations (6) and linearize with respect to perturbed quantities. Assuming 


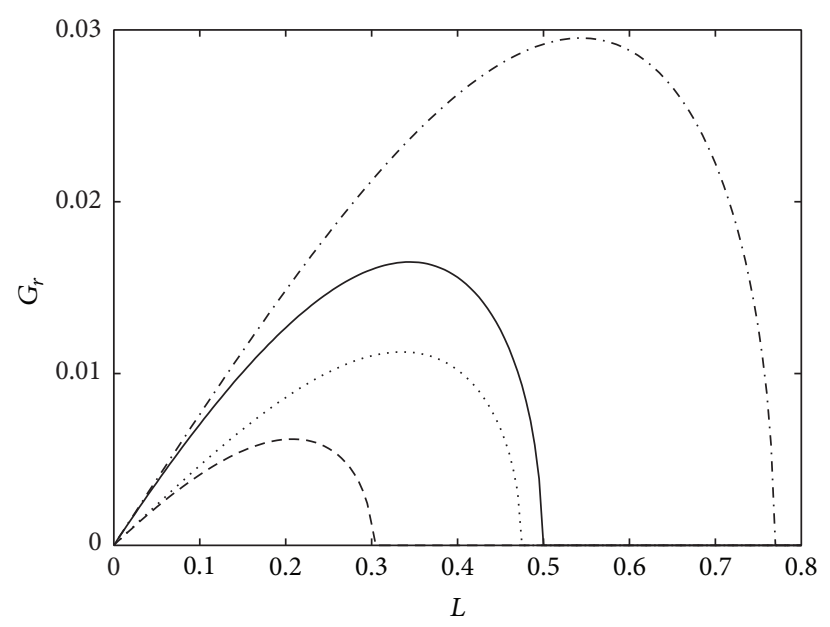

FIGURE 1: Growth rate of instability $G_{r}=\operatorname{Im}(\Omega)$ against perturbation wavenumber $L$ for $\theta=15^{\circ}$-for narrow band wave packets at fourth order: $-a_{10}=a_{20}=0.15, \cdots-a_{10}=0.15, a_{20}=0$; at third order: $\cdots-a_{10}=a_{20}=0.15, \cdots a_{1}=0.15, a_{20}=0$.

$a_{1}^{\prime}=a_{1 r}^{\prime}+i a_{1 i}^{\prime}$ and $a_{2}^{\prime}=a_{2 r}^{\prime}+i a_{2 i}^{\prime}$, where $a_{1 r}^{\prime}, a_{1 i}^{\prime}, a_{2 r}^{\prime}$, and $a_{2 i}^{\prime}$ are real, and then separating into real and imaginary parts we get four coupled equations in $a_{1 r}^{\prime}, a_{1 i}^{\prime}, a_{2 r}^{\prime}$, and $a_{2 i}^{\prime}$. Then taking Fourier transform of these four equations with respect to space variables $x$ and $y$ defined according to

$$
\widehat{f}=\frac{1}{2 \pi} \iint_{-\infty}^{\infty} f(x, y) e^{-i(L x+M y)} d x d y
$$

and assuming time dependence of the perturbed quantities $\widehat{a}_{1 r}, \widehat{a}_{1 i}, \widehat{a}_{2 r}$, and $\widehat{a}_{2 i}$ to be of the form $\exp (-i \Omega t)$, we obtain finally the following system of four equations:

$$
\begin{aligned}
& -\left(A_{+}+B_{+}\right) \hat{a}_{1 r}^{\prime}+i\left(\Omega-W_{+}-C_{+}\right) \hat{a}_{1 i}^{\prime}-D a_{20}^{2} \hat{a}_{2 r}^{\prime} \\
& \quad+i\left(E_{-}-F_{-}\right) a_{20}^{2} \hat{a}_{2 i}^{\prime}=0, \\
& \left(\Omega-W_{+}+C_{+}\right) \hat{a}_{1 r}^{\prime}-i B_{+} \hat{a}_{1 i}^{\prime}+\left(E_{+}+F_{+}\right) a_{20}^{2} \hat{a}_{2 r}^{\prime}=0, \\
& \quad-D a_{10}^{2} \hat{a}_{1 r}^{\prime}+i\left(E_{-}+F_{-}\right) a_{10}^{2} \hat{a}_{1 i}^{\prime}-\left(A_{-}+B_{-}\right) \hat{a}_{2 r}^{\prime} \\
& \quad+i\left(\Omega-W_{-}-C_{-}\right) \hat{a}_{2 i}^{\prime}=0, \\
& \left(E_{+}-F_{+}\right) a_{10}^{2} \hat{a}_{1 r}^{\prime}+\left(\Omega-W_{-}+C_{-}\right) \hat{a}_{2 r}^{\prime}-i B_{-} \hat{a}_{2 i}^{\prime}=0 .
\end{aligned}
$$

The condition of nontrivial solution of the above system gives the following biquadratic dispersion relation:

$$
\begin{aligned}
& {\left[\left(\Omega-W_{+}\right)^{2}-S_{+}\right]\left[\left(\Omega-W_{-}\right)^{2}-S_{-}\right]} \\
& =P\left(\Omega-W_{+}\right)\left(\Omega-W_{-}\right)-Q_{+}\left(\Omega-W_{+}\right) \\
& \quad-Q_{-}\left(\Omega-W_{-}\right)+R .
\end{aligned}
$$

The coefficients $A_{ \pm}, B_{ \pm}, C_{ \pm}, D, E_{ \pm}, F_{ \pm}, P, Q_{ \pm}, R, S_{ \pm}$, and $W_{ \pm}$ are given in Appendix B. The subscripts $( \pm)$ are used just for convenience of writing the coefficients.

We have plotted growth rate of instability $G_{r}=\operatorname{Im}(\Omega)$ both for unidirectional and bidirectional perturbations. In Figures 1-4, growth rate of instability $G_{r}$ is plotted against
$L, L$ being perturbation wavenumber along $x$-direction. In Figure 1, it is observed that the growth rate of instability for two obliquely interacting wave packets is much higher than that for a single wave packet. This is true at third order and also at fourth order. Figure 2 shows that there is an increase in the growth rate of instability of one wave packet when the amplitude of the other wave packet increases. Figure 3 shows a comparison between the growth rate of instability for narrow band wave packets and that of broader bandwidth wave packets. From Figure 3 it is revealed that the growth rate of instability in crossing sea states is much higher than that for a single wave packet having broader bandwidth. In Figure 4 we can observe the variation in $G_{r}$ with respect to $\theta$ for broader bandwidth wave packets when $a_{10}=0.18$ and $a_{20}=0.15$. The figure shows that the growth rate of instability is higher when the angle of interaction between the two wave packets is smaller.

Solving the dispersion relation (15) numerically, we have plotted instability growth rate curves in perturbed wavenumber plane. In Figures 5, 6, 7, and 8, we have shown contour plots for instability growth rate for bidirectional perturbations. In Figures 5-7, amplitudes of the two uniform wave trains are considered equal, while in Figure 8 , the amplitudes are different. It is known from the investigations of Trulsen and Dysthe [10] and Debsarma and Das [12, 17] that the stability analysis made from higher order evolution equation for broader bandwidth wave packets gives more accurate results. Comparing the upper row figures with the corresponding lower row figures in Figures 5-8, we see that there is little shrinkage in the instability region for broader bandwidth wave packets.

\section{Conclusion}

Keeping in view the importance of higher order evolution equations valid for broad band wave packets, we have derived here two coupled nonlinear evolution equations for broader bandwidth surface gravity wave packets under the crossing 


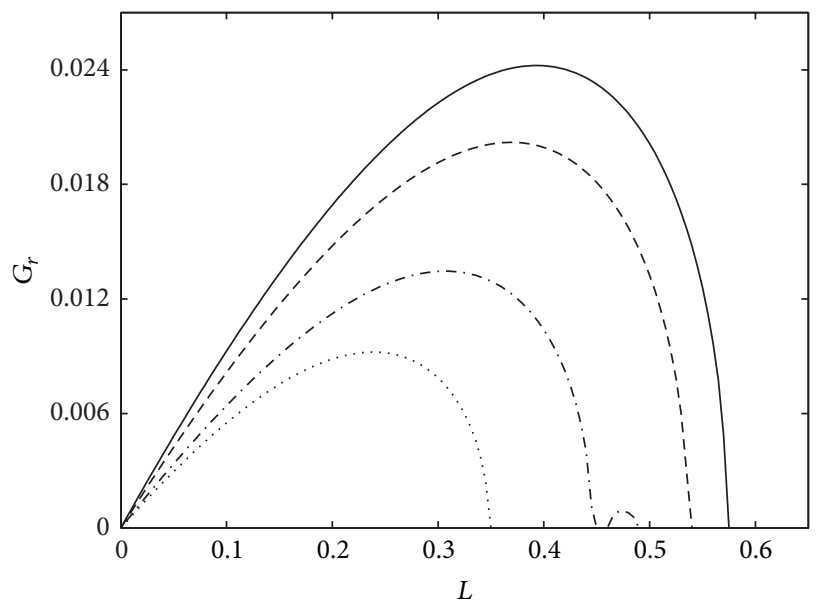

FIGURE 2: Growth rate of instability $G_{r}=\operatorname{Im}(\Omega)$ against perturbation wavenumber $L$ at fourth order for narrow band wave packets, $\theta=15^{\circ}$, $a_{10}=0.2,-a_{20}=0.2, \cdots-a_{20}=0.1, \cdots \cdots a_{20}=0.075, \cdots a_{20}=0$.

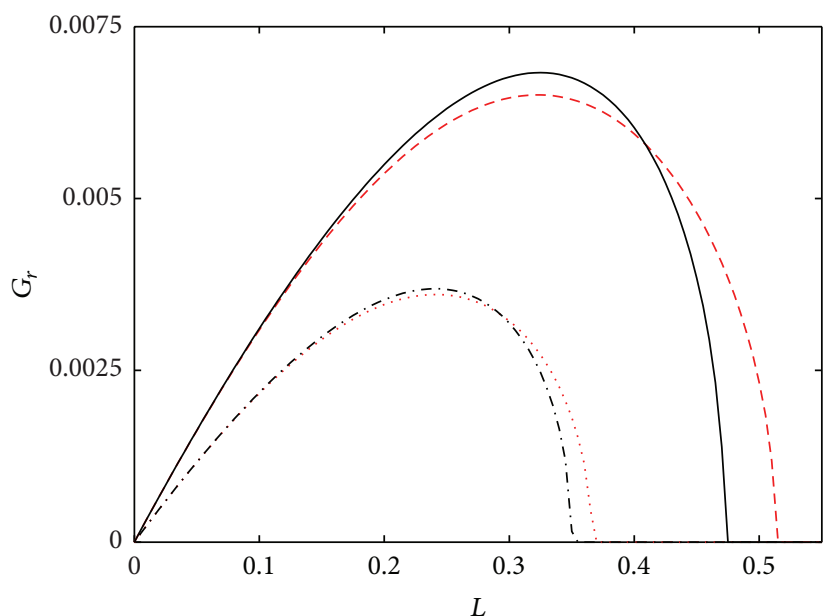

FIGURE 3: Growth rate of instability $G_{r}=\operatorname{Im}(\Omega)$ against perturbation wavenumber $L$ for $\theta=28^{\circ}$ - for narrow band wave packets: $-a_{10}=$ $a_{20}=0.12, \cdots-a_{10}=0.12, a_{20}=0$; for broad band wave packet: $-\cdots a_{10}=a_{20}=0.12, \cdots a_{10}=0.12, a_{20}=0$.

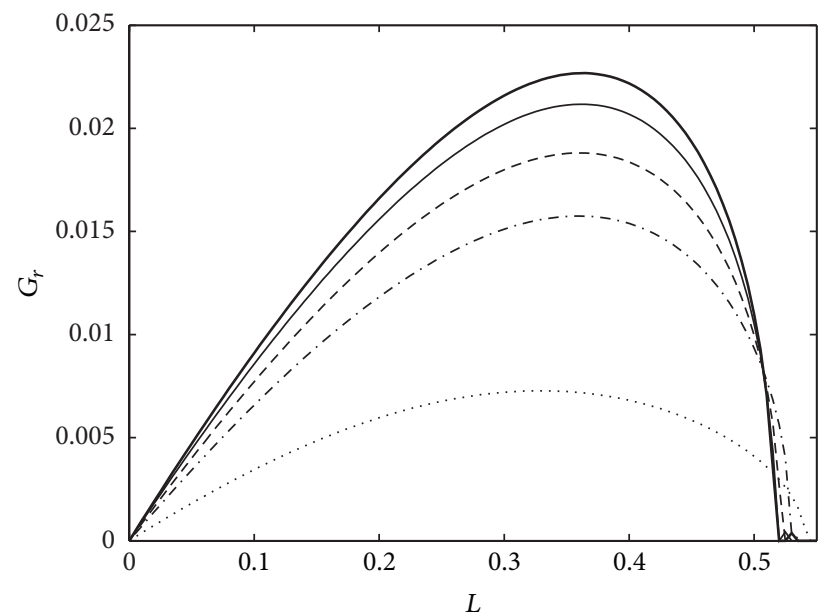

FIGURE 4: Growth rate of instability $G_{r}=\operatorname{Im}(\Omega)$ against perturbation wavenumber $L$ for broad band wave packets when $a_{10}=0.18$ and $a_{20}=0.15:-\theta=5^{\circ},-\theta=10^{\circ}, \cdots-\theta=15^{\circ}, \cdots-\theta=20^{\circ}, \cdots \theta=30^{\circ}$. 

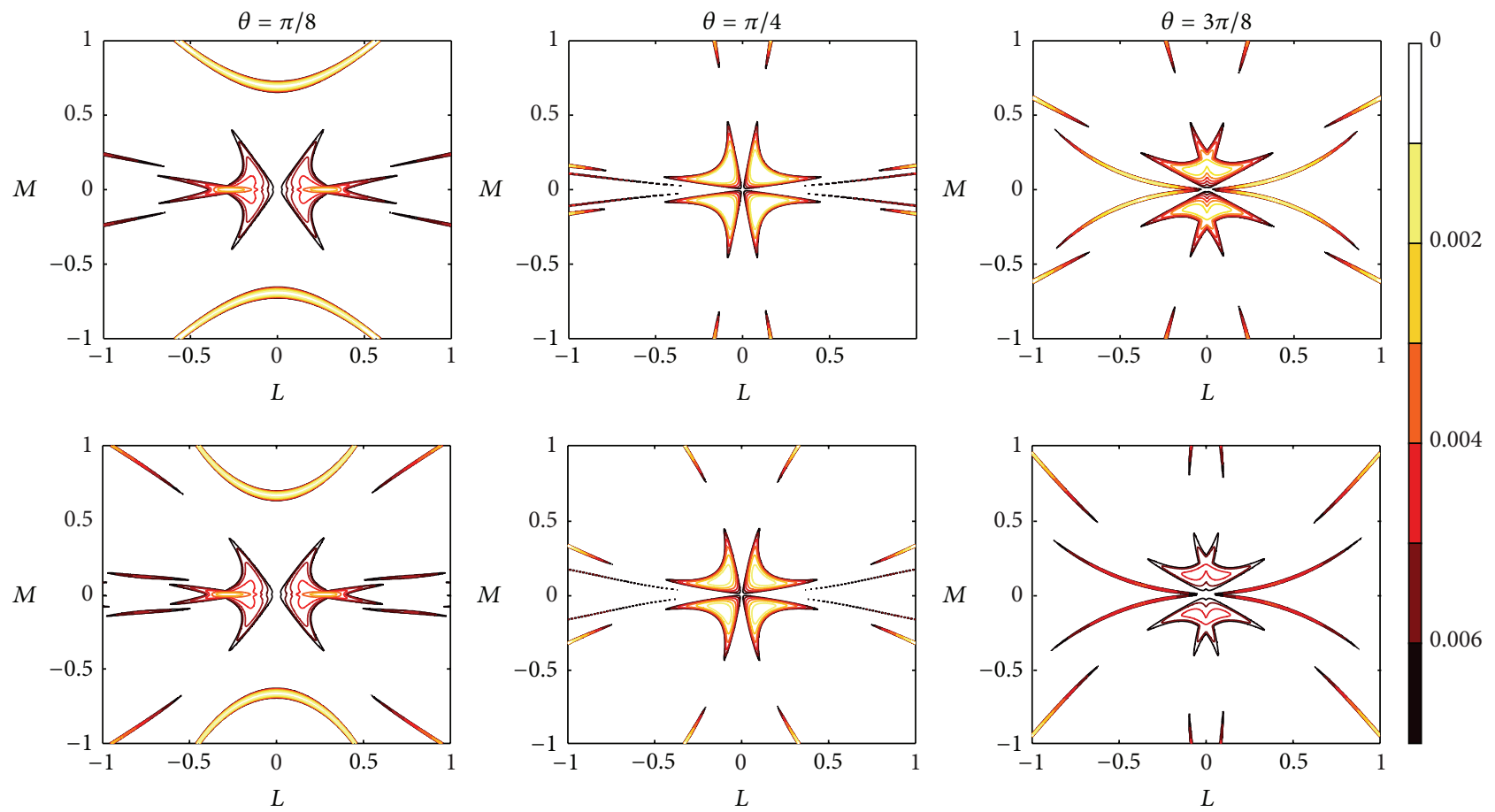

FIGURE 5: Growth rate of instability $G_{r}=\operatorname{Im}(\Omega)$ in perturbed wavenumber plane, $a_{10}=a_{20}=0.1$. Figures in the upper row are for narrow band wave packets; figures in the lower row are for broad band wave packets.
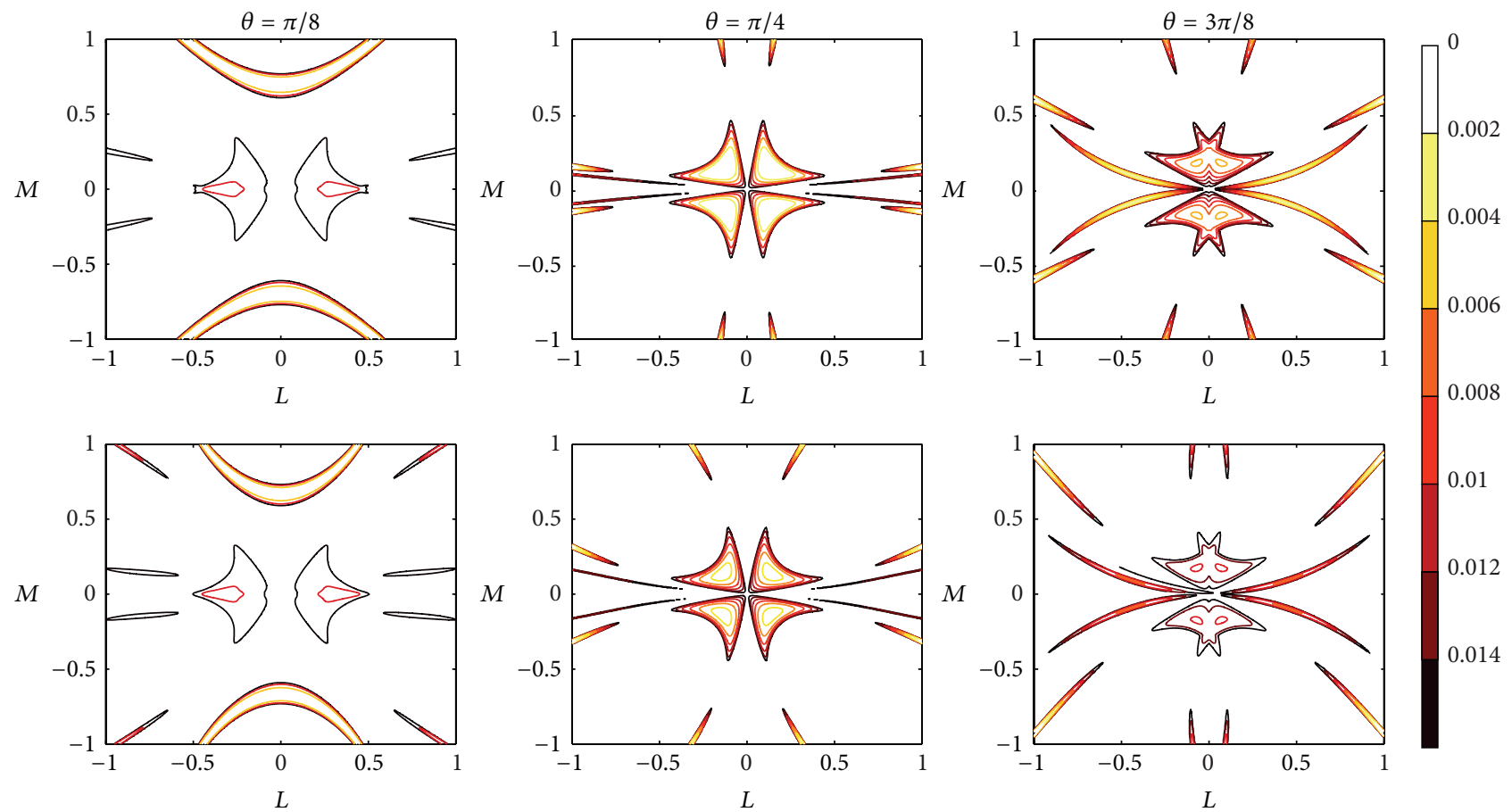

FIGURE 6: Growth rate of instability $G_{r}=\operatorname{Im}(\Omega)$ in perturbed wavenumber plane, $a_{10}=a_{20}=0.15$. Figures in the upper row are for narrow band wave packets; figures in the lower row are for broad band wave packets. 

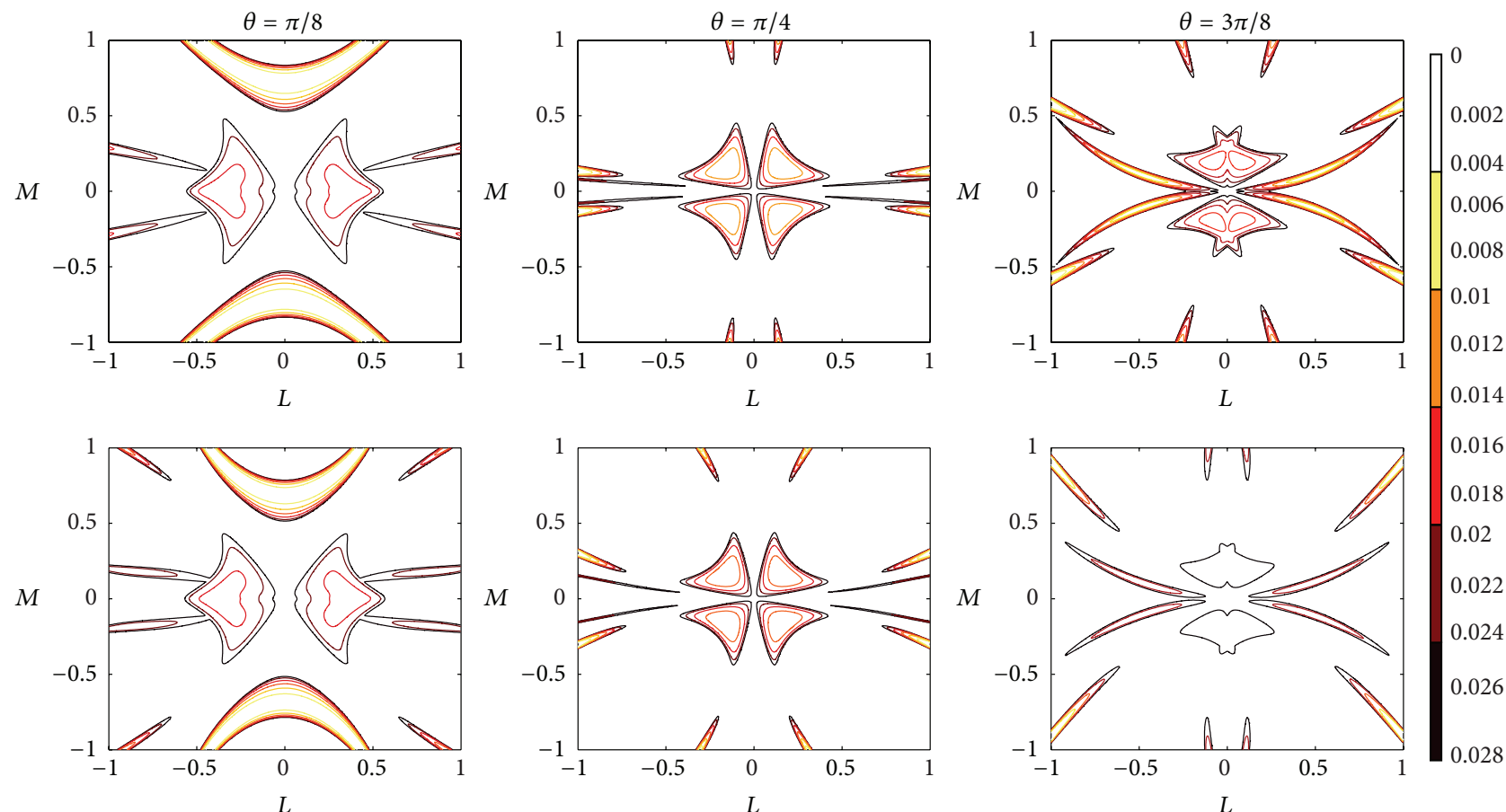

FIGURE 7: Growth rate of instability $G_{r}=\operatorname{Im}(\Omega)$ in perturbed wavenumber plane, $a_{10}=a_{20}=0.2$. Figures in the upper row are for narrow band wave packets; figures in the lower row are for broad band wave packets.
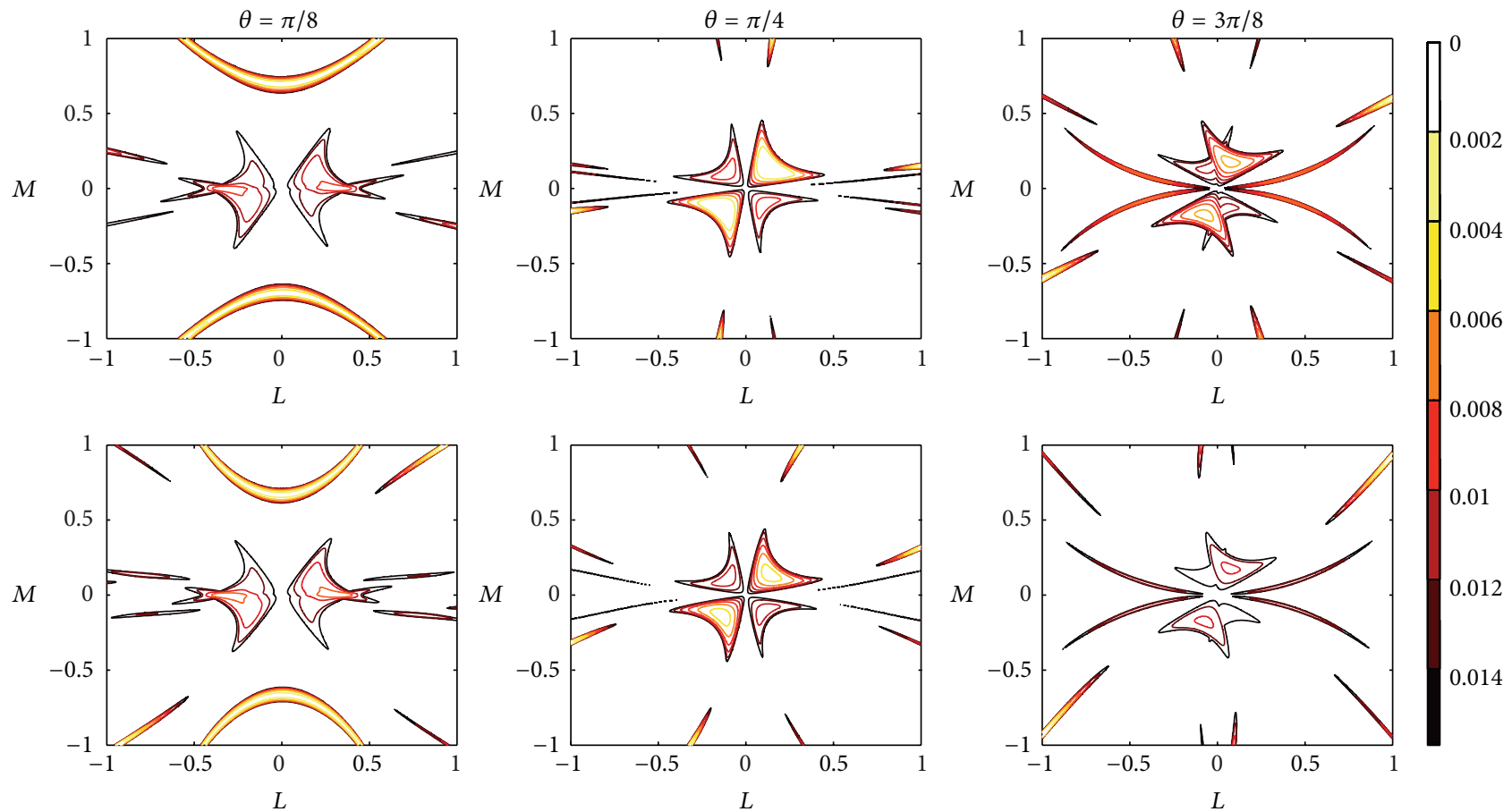

FIGURE 8: Growth rate of instability $G_{r}=\operatorname{Im}(\Omega)$ in perturbed wavenumber plane, $a_{10}=0.15, a_{20}=0.1$. Figures in the upper row are for narrow band wave packets; figures in the lower row are for broad band wave packets. 
sea states assumption. The evolution equations derived here in crossing sea states can be considered as an extension of the evolution equation for a single broader bandwidth wave packet derived by Trulsen and Dysthe [10]. Using the evolution equations derived here we have also carried out stability analysis of two obliquely interacting uniform wave trains. Results obtained here are in agreement with the corresponding results of Shukla et al. [6] at third order with narrow band wave assumption. Modifications in the instability growth rates with the assumption of broader bandwidth wave packets are shown graphically in this paper. It is found that the growth rate of instability in a situation of crossing sea states is much higher than that for a single wave packet even for broader bandwidth wave packets. So, generation of freak waves due to nonlinear interaction of broader bandwidth wave packets in crossing sea states is confirmed. It is also found that the growth rate of instability is higher when the angle of interaction between the two wave packets is smaller. Comparing the stable-unstable regions for broader bandwidth wave packets with those for narrow bandwidth wave packets, we see that there is little shrinkage in the instability region for broader bandwidth wave packets.

\section{Appendices}

\section{A. Coefficients of the Evolution Equations}

Consider

$$
\begin{aligned}
& \beta_{1}=\frac{k}{2 k_{c}}, \quad \beta_{2}=\frac{l}{2 k_{c}}, \quad \beta_{3}=\frac{2 l^{2}-k^{2}}{8 k_{c}^{2}}, \\
& \beta_{4}=-\frac{3 k l}{4 k_{c}^{2}}, \quad \beta_{5}=\frac{2 k^{2}-l^{2}}{8 k_{c}^{2}}, \\
& \beta_{6}=\frac{6 k l^{2}-k^{3}}{16 k_{c}^{3}}, \quad \beta_{7}=\frac{6 l^{3}-15 k^{2} l}{16 k_{c}^{3}}, \\
& \beta_{8}=\frac{6 k^{3}-15 k l^{2}}{16 k_{c}^{3}}, \quad \beta_{9}=\frac{6 k^{2} l-l^{3}}{16 k_{c}^{3}}, \\
& \beta_{10}=\frac{5 k^{4}-60 k^{2} l^{2}+12 l^{4}}{128 k_{c}^{4}}, \quad \beta_{11}=\frac{35 k^{3} l-42 k l^{3}}{32 k_{c}^{4}} \\
& \beta_{12}=\frac{-30 k^{4}+171 k^{2} l^{2}-30 l^{4}}{64 k_{c}^{4}}, \quad \beta_{13}=\frac{-42 k^{3} l+35 k l^{3}}{32 k_{c}^{4}} \\
& \beta_{17}=\frac{-70 k^{5}+707 k^{3} l^{2}-378 k l^{4}}{128 k_{c}^{5}}, \\
& \beta_{16}=\frac{12 k^{4}-60 k^{2} l^{2}+5 l^{4}}{128 k_{c}^{4}}, \\
& \beta_{15}=\frac{7 k^{5}-140 k^{3} l^{2}+84 k l^{4}}{25 k_{c}^{5}},
\end{aligned}
$$$$
\beta_{18}=\frac{-70 l^{5}+707 k^{2} l^{3}-378 k^{4} l}{128 k_{c}^{5}},
$$$$
\beta_{19}=\frac{84 k^{5}-756 k^{3} l^{2}+315 k l^{4}}{256 k_{c}^{5}}
$$$$
\beta_{20}=\frac{7 l^{5}-140 k^{2} l^{3}+84 k^{4} l}{256 k_{c}^{5}}
$$$$
\lambda_{1}=\frac{1}{2}, \quad \lambda_{2}=-\frac{3 k}{2 k_{c}}, \quad \lambda_{3}=-\frac{3 l}{2 k_{c}},
$$$$
\lambda_{4}=-\frac{k}{4 k_{c}}, \quad \lambda_{5}=-\frac{l}{4 k_{c}}
$$$$
\lambda_{6}=\frac{1}{2}\left(1+\frac{k^{2}}{k_{c}^{2}}\right), \quad \lambda_{7}=\frac{1}{2}\left(1+\frac{l^{2}}{k_{c}^{2}}\right), \quad \lambda_{8}=\frac{k l}{k_{c}^{2}},
$$$$
\mu_{1}=\frac{1}{k_{c}^{4}\left(k-2 k_{c}\right)}\left[k^{5}-k^{3} l^{2}-3 k l^{4}-2 k_{c}\left(k^{4}-k^{2} l^{2}-l^{4}\right)\right] \text {, }
$$$$
\mu_{2}=-\frac{1}{4 k_{c}^{5}\left(k-2 k_{c}\right)^{2}}\left[30 k^{7}+54 k^{5} l^{2}+18 k^{3} l^{4}-8 k l^{6}\right.
$$$$
\left.-8 k_{c}\left(3 k^{6}+3 k^{4} l^{2}-l^{6}\right)\right]
$$$$
\mu_{3}=-\frac{k}{2 k_{c}^{5}\left(k-2 k_{c}\right)}\left[k^{5}-k^{3} l^{2}-3 k l^{4}\right.
$$$$
\left.-2 k_{c}\left(k^{4}-k^{2} l^{2}-l^{4}\right)\right],
$$$$
\mu_{4}=\frac{1}{2 k_{c}^{5}\left(k-2 k_{c}\right)^{2}}\left[\left(15 k^{6} l+45 k^{4} l^{3}+41 k^{2} l^{5}+12 l^{7}\right)\right.
$$$$
\left.-4 k_{c}\left(3 k^{5} l+9 k^{3} l^{3}+5 k l^{5}\right)\right],
$$$$
\mu_{5}=\frac{1}{2 k_{c}^{5}\left(k-2 k_{c}\right)}\left[2 k^{5} l+10 k^{3} l^{3}+7 k l^{5}\right.
$$$$
\left.-k_{c}\left(4 k^{4} l+10 k^{2} l^{3}+6 l^{5}\right)\right],
$$$$
\mu_{6}=-\frac{1}{2 k_{c}^{5}\left(k-2 k_{c}\right)^{2}}\left[10 k^{6} l+6 k^{4} l^{3}-9 k^{2} l^{5}-4 l^{7}\right.
$$$$
\left.-2 k_{c}\left(4 k^{5} l+k^{3} l^{3}-4 k l^{5}\right)\right],
$$$$
\mu_{7}=\frac{k^{2}}{2 k_{c}^{2}}, \quad \mu_{8}=-\frac{l^{2}}{2 k_{c}^{2}} \text {. }
$$

\section{B. Coefficients of the Dispersion Relation (15)}

Consider

$$
\begin{aligned}
& A_{+}=\left[2 \lambda_{1}-\frac{2\left(\lambda_{6} L^{2}+\lambda_{7} M^{2}+\lambda_{8} L M\right)}{\sqrt{L^{2}+M^{2}}}\right] a_{10}^{2}, \\
& A_{-}=\left[2 \lambda_{1}-\frac{2\left(\lambda_{6} L^{2}+\lambda_{7} M^{2}-\lambda_{8} L M\right)}{\sqrt{L^{2}+M^{2}}}\right] a_{20}^{2},
\end{aligned}
$$




$$
\begin{aligned}
& B_{ \pm}=\beta_{3} L^{2} \pm \beta_{4} L M+\beta_{5} M^{2} \\
& -\left(\beta_{10} L^{4} \pm \beta_{11} L^{3} M+\beta_{12} L^{2} M^{2} \pm \beta_{13} L M^{3}+\beta_{14} M^{4}\right), \\
& C_{+}=\left(\lambda_{4} L+\lambda_{5} M\right) a_{10}^{2}, \quad C_{-}=\left(\lambda_{4} L-\lambda_{5} M\right) a_{20}^{2} \\
& D=2 \mu_{1}-\frac{2 \mu_{7} L^{2}+2 \mu_{8} M^{2}}{\sqrt{L^{2}+M^{2}}}, \\
& E_{ \pm}=\left(\mu_{2} \pm \mu_{3}\right) L, \quad F_{ \pm}=\left(\mu_{5} \pm \mu_{6}\right) M, \\
& W_{+}=\beta_{1} L+\beta_{2} M-\beta_{6} L^{3}-\beta_{7} L^{2} M-\beta_{8} L M^{2} \\
& -\beta_{9} M^{3}+\beta_{15} L^{5}+\beta_{16} L^{4} M+\beta_{17} L^{3} M^{2} \\
& +\beta_{18} L^{2} M^{3}+\beta_{19} L M^{4}+\beta_{20} M^{5} \\
& -\left(\lambda_{2} L+\lambda_{3} M\right) a_{10}^{2}-\left(\mu_{2} L+\mu_{4} M\right) a_{20}^{2} \\
& W_{-}=\beta_{1} L-\beta_{2} M-\beta_{6} L^{3}+\beta_{7} L^{2} M \\
& -\beta_{8} L M^{2}+\beta_{9} M^{3}+\beta_{15} L^{3}-\beta_{16} L^{4} M+\beta_{17} L^{3} M^{2} \\
& -\beta_{18} L^{2} M^{3}+\beta_{19} L M^{4}-\beta_{20} M^{5} \\
& -\left(\lambda_{2} L-\lambda_{3} M\right) a_{20}^{2}-\left(\mu_{2} L-\mu_{4} M\right) a_{10}^{2}, \\
& S_{ \pm}=B_{ \pm}^{2}+B_{ \pm} A_{ \pm}+C_{ \pm}^{2}, \\
& P=\left(E_{+}^{2}+E_{-}^{2}-F_{+}^{2}-F_{-}^{2}\right) a_{10}^{2} a_{20}^{2}, \\
& Q_{+}=\left[C_{-}\left(E_{+}^{2}-E_{-}^{2}-F_{+}^{2}+F_{-}^{2}\right)\right. \\
& \left.+D B_{-}\left(E_{+}+E_{-}+F_{+}+F_{-}\right)\right] a_{10}^{2} a_{20}^{2} \text {, } \\
& Q_{-}=\left[C_{+}\left(E_{+}^{2}-E_{-}^{2}-F_{+}^{2}+F_{-}^{2}\right)\right. \\
& \left.+D B_{+}\left(E_{+}+E_{-}-F_{+}-F_{-}\right)\right] a_{10}^{2} a_{20}^{2}, \\
& R=\left[C_{+} C_{-}\left(E_{+}^{2}+E_{-}^{2}-F_{+}^{2}-F_{-}^{2}\right)\right. \\
& +C_{+} D B_{-}\left(E_{+}-E_{-}+F_{+}-F_{-}\right) \\
& +C_{-} D B_{+}\left(E_{+}-E_{-}-F_{+}+F_{-}\right) \\
& +B_{+}\left(B_{-}+A_{-}\right)\left(E_{+}-F_{+}\right)\left(E_{-}-F_{-}\right) \\
& +B_{-}\left(B_{+}+A_{+}\right)\left(E_{+}+F_{+}\right)\left(E_{-}+F_{-}\right) \\
& \left.+B_{+} B_{-} D^{2}\right] a_{10}^{2} a_{20}^{2}-\left(E_{+}^{2}-F_{+}^{2}\right)\left(E_{-}^{2}-F_{-}^{2}\right) a_{10}^{4} a_{20}^{4}
\end{aligned}
$$

\section{Conflict of Interests}

The authors declare that there is no conflict of interests regarding the publication of this paper.

\section{References}

[1] C. Kharif and E. Pelinovsky, "Physical mechanisms of the rogue wave phenomenon," European Journal of Mechanics B: Fluids, vol. 22, no. 6, pp. 603-634, 2003.

[2] A. Toffoli, J. Monbaliu, J. M. Lefěvre, and E. Bitner-Gregersen, "Dangerous sea-states for marine operations," in Proceedings of the 14th International Offshore and Polar Engineering Conference (ISOPE '04), pp. 85-92, Toulon,France, May 2004.

[3] K. B. Dysthe, "Modelling a "rogue wave"-speculations or a realistic possibility ?" in Proceedings of the Rogue Waves, M. Olagon and G. Athanassoulis, Eds., pp. 255-264, Ifremer, France, 2002.

[4] B. S. White and B. Fornberg, "On the chance of freak waves at sea," Journal of Fluid Mechanics, vol. 355, pp. 113-138, 1998.

[5] M. Onorato, A. R. Osborne, and M. Serio, "Modulational instability in crossing sea states: a possible mechanism for the formation of freak waves," Physical Review Letters, vol. 96, no. 1, Article ID 014503, 2006.

[6] P. K. Shukla, I. Kourakis, B. Eliasson, M. Marklund, and L. Stenflo, "Instability and evolution of nonlinearly interacting water waves," Physical Review Letters, vol. 97, no. 9, Article ID 094501, 2006.

[7] F. E. Laine-Pearson, "Instability growth rates of crossing sea states," Physical Review E, vol. 81, Article ID 036316, pp. 1-7, 2010.

[8] K. B. Dysthe, "Note on a modification to the nonlinear Schrödinger equation for application to deep water waves," Proceedings of the Royal Society of London A, vol. 369, pp. 105114, 1979.

[9] O. Gramstad and K. Trulsen, "Fourth-order coupled nonlinear Schrödinger equations for gravity waves on deep water," Physics of Fluids, vol. 23, no. 6, Article ID 062102, 2011.

[10] K. Trulsen and K. B. Dysthe, "A modified nonlinear Schrödinger equation for broader bandwidth gravity waves on deep water," Wave Motion, vol. 24, no. 3, pp. 281-289, 1996.

[11] J. W. McLean, Y. C. Ma, D. U. Martin, P. G. Saffman, and H. C. Yuen, "Three-dimensional instability of finite-amplitude water waves," Physical Review Letters, vol. 46, no. 13, pp. 817-820, 1981.

[12] S. Debsarma and K. P. Das, "A higher-order nonlinear evolution equation for broader bandwidth gravity waves in deep water," Physics of Fluids, vol. 17, no. 10, Article ID 104101, 2005.

[13] T. B. Benjamin and P. J. Olver, "A higher order nonlinear evolution equation for broader bandwidth gravity waves in deep water," Journal of Fluid Mechanics, vol. 125, pp. 137-185, 1982.

[14] V. E. Zakharov, "Stability of periodic waves of finite amplitude on the surface of a deep fluid," Journal of Applied Mechanics and Technical Physics, vol. 9, no. 2, pp. 190-194, 1968.

[15] V. P. Krasitskii, "On reduced equations in the Hamiltonian theory of weakly nonlinear surface waves," Journal of Fluid Mechanics, vol. 272, pp. 1-21, 1994.

[16] S. S. Debsarma and K. P. Das, "Fourth-order nonlinear evolution equations for a capillary-gravity wave packet in the presence of another wave packet in deep water," Physics of Fluids, vol. 19, no. 9, Article ID 097101, 2007.

[17] S. Debsarma and K. P. Das, "A higher-order nonlinear evolution equation for broader bandwidth gravity waves in deep water," Physics of Fluids, vol. 17, no. 10, Article ID 104101, 2005. 

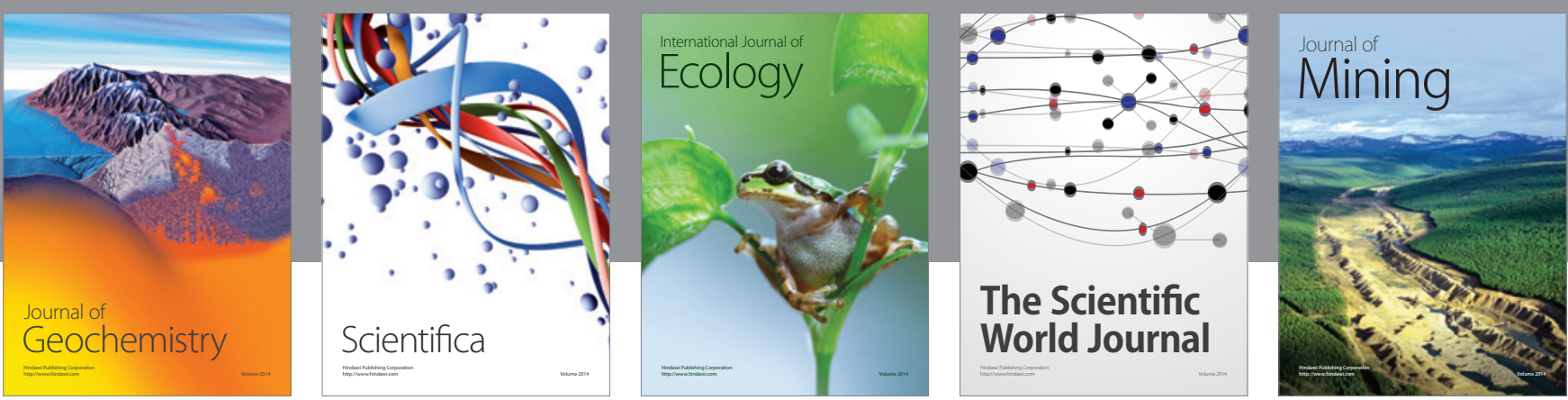

The Scientific World Journal
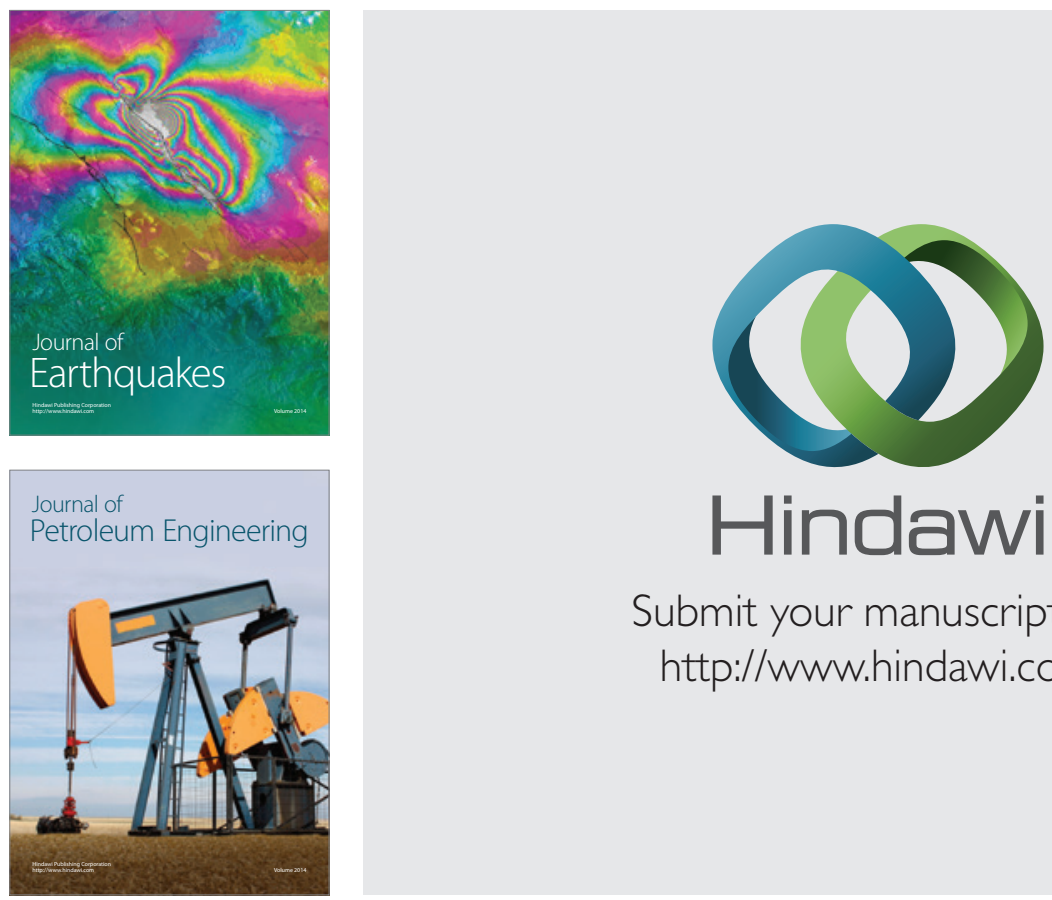

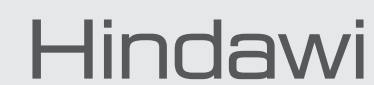

Submit your manuscripts at

http://www.hindawi.com
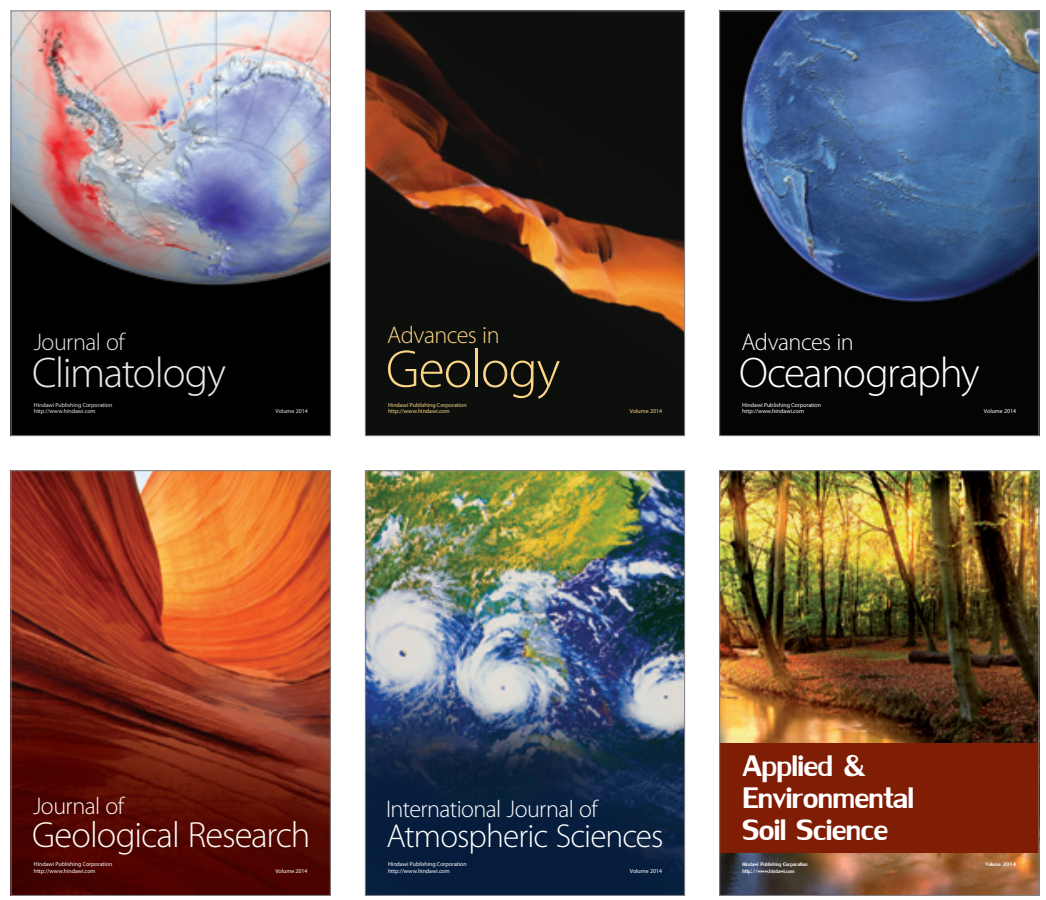
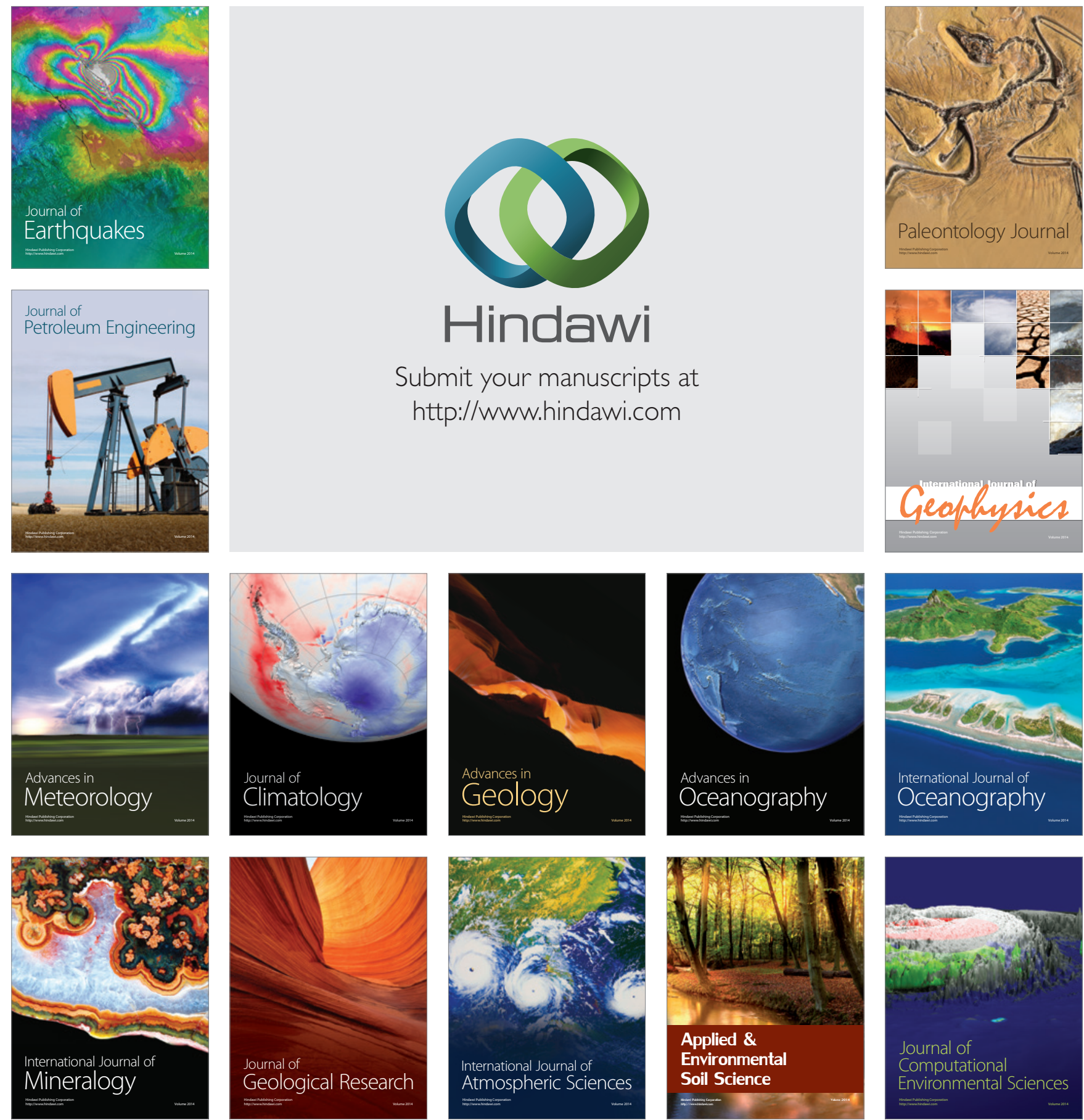